\title{
HOW TO WRITE AN ABSTRACT: The
}

\section{Concise Approach?}

\section{By}

\author{
Mala Babagana Gutti
}

B.Eng. Student, Department of Civil and Water Resources Engineering,

University of Maiduguri, Maiduguri, Nigeria

Email: malabgutti@gmail.com

$20^{\text {th }}$ January, 2019 


\section{Introduction}

An abstract is a short summary of your completed research. If done well, it makes the reader want to learn more about your research. It is a condensed version or summary of your research study and also a means of conveying what was done and why, what was found, and the implications. An abstracts should be;

- Complete: It should cover the major parts of the article, study, or project.

- Concise: It should not contain excess unnecessary information.

- Clear: It should be readable, well organized, and meaningful.

- Cohesive: The sentences should flow smoothly between the parts.

- Professional: Limit each abstract to a single paragraph that identifies the content it is describing and only use abbreviations when they have been defined in the opening statement or acronyms if they are understood by a wide audience.

\section{Types of Abstracts}

The word abstract comes from the Latin abstractum, which means a condensed form of a longer piece of writing. The type of abstract you write depends on your discipline area. There are two main types of abstract.;

1. Descriptive abstracts: Descriptive abstracts are generally used for humanities and social science papers or psychology essays. This type of abstract is usually very short (50-100 words). Most descriptive abstracts have certain key parts in common. They are:

- Background.

- Purpose. 
- particular interest/focus of paper.

- overview of contents (not always included).

2. Informative abstracts: Informative abstracts are generally used for science, engineering or psychology reports. You must get the essence of what your report is about, usually in about 200 words. Most informative abstracts also have key parts in common. Each of these parts might consist of 1-2 sentences. The parts include:

- Background.

- Aim or purpose of research.

- Method used.

- Findings/results.

- Conclusion.

\section{Qualities of a good Abstract}

1. Includes one or more coherent and concise paragraphs (usually 200-300 words).

2. Uses an introduction-body-conclusion structure in which the parts of the report are discussed in order: purpose, research questions, methods, findings, conclusions, recommendations.

3. Adds no new information just merely summarizes.

4. Can be understood without reading the paper.

5. Provides a condensed and concentrated version of the full text.

6. Does not contain citations.

7. Does not contain lengthy background information. 
8. Does not contain any sort of illustration, figure, or table, or references to them.

\section{How to draft an Abstract?}

Since an abstract summarizes, in one paragraph (usually 200-300 words), the major aspects of an entire paper. A good trick is to plan your argument in 6 sentences, and then use these to structure your abstract;

$\checkmark$ Introduction: Describe what topic your paper covers and provide the reader with a background to the study and also avoid unnecessary content.

$\checkmark$ State the problem: What is the key research question or problem? Again, in one sentence.

$\checkmark$ Summarize why nobody else has adequately answered the research question yet: Emphasize the gap in the literature. You could use a phrase such as "Previous work has failed to address...".

$\checkmark$ Explain how you have approached the research question: What's your new idea or solution to the problem identified?

$\checkmark$ In one sentence, describe how you went about doing the research: Provide an outline of the methods or procedure you used. Did you run experiments? Carry out case studies? Interviews? Etc. in summary.

$\checkmark$ What is the key impact of your research: What conclusions did you draw and what are the implications? 


\section{The Components of an Abstract}

These are the basic components of an abstract in any discipline:

1. Introduction/Background of the study: What is the background of your study? What does it cover? It should explain why your abstract is important or novel, provide the context or explanation for doing the study not the whole history but the current situation, what is already known about the subject? What is not known, and hence what you intend to examine? Should state the aim of the study, what are you hoping to find out or what is your hypothesis?

2. Motivation/problem statement: Why do we care about the problem? What practical, scientific, theoretical or artistic gap is your research filling?

3. Methods/procedure/approach: What did you actually do to get your results? (e.g. Interview or experiments etc.). Specific population studied include sampling frames and response rates when appropriate, how many people were approached, how many participated? And in the case of quantitative or qualitative methods, specific statistical analyses conducted, measures and outcomes explored, time from and duration of the study should be stated.

4. Results/findings/product: As a result of completing the above procedure, what did you learn/invent/create? It should concisely describe how your results pertain to your study aim or hypothesis. And also describe your main findings with data if available.

5. Conclusion/implications: What are the larger implications of your findings, especially for the problem/gap identified in step 1? Conclusions should be reasonable and supported by findings. It should also explain your main findings and why they are 
important. However, it's important to note that the weight accorded to the different components can vary by discipline.

\section{What to Include in an Abstract?}

Perry et al (2003) recommend that an abstract should include the following seven elements:

Element 1: The abstract has to start with a brief theme sentence to orientate the reader about the overall issue addressed in the thesis. The sentence should grab the reader's attention.

Element 2: The abstract should then indicate the main aim or purpose of the study.

Element 3: Next, the academic and/or practical importance of the study should be explained.

Element 4: The methodology used in the study should also be described.

Element 5: The main findings of the study should be summarized.

Element 6: Statements of conclusions should indicate the contribution made by the study in filling gaps in the literature.

Element 7: Finally, the practical or managerial implications of the study's findings should be highlighted where appropriate (highly applicable for social science studies). 


\section{REFERENCES}

[1] Karis Moxley (2015). "How to Write an Abstract" Writing Lab, University of Cape Town

[2] Dr. K's Short and Snappy (2008) "How to Write an Abstract “ by Jo Koster Copyrights, Department of English, Winthrop University, kosterj@winthrop.edu

[3] Leah Carroll (n.d) "HOW TO WRITE AN ABSTRACT: Tips and Samples" Ph.D., Director, Office of Undergraduate Research

[4] Duke University Press (n.d) "Writing Abstracts and Keywords" URL: http://www.dukeupress.edu

[5] Scientific Program Committee (2016) "How to write an Abstract" STD Prevention Conference

[6] Sandeep B. Bavdekar and Nithya J. Gogtay (2015). ”Writing an Abstract for a Research Manuscript: Providing an Honest, Succinct and Complete Summary" Journal of The Association of Physicians of India: Vol. 63. Dec, 2015. URL: https://www.researchgate.net/publication/286862049

[7] Writing Centre Learning Guide (2014). "Writing an Abstract” Copyright of the University of Adelaide, 2014.

[8] Perry, C., Carson, D. \& Gilmore, A. (2003). “Joining a conversation: Writing for EJM's editors, reviewers and readers required planning, care and persistence.” European Journal of Marketing, Vol. 37, Issue (5/6): pp625-557.

[9] Students Guide Committee (n.d). "Writing an abstract: Understanding and developing abstracts" the University of Melbourne URL: $\underline{\text { http://www.services.unimelb.edu.au/academicskills }}$ 